\title{
Survival amplitude, instantaneous energy and decay rate of an unstable system: Analytical results
}

\author{
K. Raczyńska, K. Urbanowski ${ }^{\dagger}$ \\ University of Zielona Góra, Institute of Physics, \\ ul. Prof. Z. Szafrana 4a, 65-516 Zielona Góra, Poland.
}

November 9, 2018

\begin{abstract}
We consider a model of a unstable state defined by the truncated Breit-Wigner energy density distribution function. An analytical form of the survival amplitude $a(t)$ of the state considered is found. Our attention is focused on the late time properties of $a(t)$ and on effects generated by the non-exponential behavior of this amplitude in the late time region: In 1957 Khalfin proved that this amplitude tends to zero as $t$ goes to the infinity more slowly than any exponential function of $t$. This effect can be described using a time-dependent decay rate $\gamma(t)$ and then the Khalfin result means that this $\gamma(t)$ is not a constant but at late times it tends to zero as $t$ goes to the infinity. It appears that the energy $E(t)$ of the unstable state behaves similarly: It tends to the minimal energy $E_{\text {min }}$ of the system as $t \rightarrow \infty$. Within the model considered we find two first leading time dependent elements of late time asymptotic expansions of $E(t)$ and $\gamma(t)$. We discuss also possible implications of such a late time asymptotic properties of $E(t)$ and $\gamma(t)$ and cases where these properties may manifest themselves.
\end{abstract}

PACS: 03.65.-w, 11.10.St, 95.36.+x

${ }^{*} \mathrm{e}-\mathrm{mail}$ : yaraczynska@gmail.com

${ }^{\dagger} \mathrm{e}-$ mail: K.Urbanowski@if.uz.zgora.pl 


\section{Introduction}

Attempts to describe time evolution of unstable states within the Quantum Mechanics were made practically from times when this theory started to be born. The most known result from these times is the Weisskopf-Wigner theory od spontaneous emission [1]. Considering the excited atomic levels and applying the Shrödinger equation to describe the time evolution Weisskopf and Wigner found that to a good approximation the non-decay probability of the exited levels is a decreasing function of time having an exponential form [1]. Further theoretical studies of the quantum decay process showed that basic principles of the quantum theory does not allow it to be described by an exponential decay law at very late times [2, 3] and at initial stage of the decay process (see [3] and references therein). Theoretical analysis shows that at late times the survival probability (i. e. the decay law) should tends to zero as $t \rightarrow \infty$ much more slowly than any exponential function of time and that as function of time it has the inverse power-like form at this regime of time [2, 3]. There was many unsuccessful attempts to verify experimentally predicted deviations from the exponential form of the decay law at late times regime (see eg. [4]). The first experimental evidence of these deviations at long time regime was reported in [5]. Rothe and his group preparing their experiment used successfully conclusions resulting from theoretical studies of models of unstable states and conditions leading to the non-exponential behavior of the survival amplitude (see, e.g., [6] - [10]). The result reported by Rhote's group gives rise to another important problem: If (and how) the late time deviations from the exponential decay affect the energy of the unstable state and its decay rate. Theoretical studies of models of unstable states can bring us closer to understanding and explaining this problem. This paper contains analysis of the quantum unstable system modeled by the Breit-Wigner energy density distribution function. Studies of such models are known in the literature but usually these studies were limited to the analysis of properties of the survival amplitude (see, eg. [11] and [12, 13]). The first leading late time terms of asymptotic series expansions for the energy $E(t)$ and decay rate $\gamma(t)$ of the unstable state were found within such a model in [12, 13]. In this paper we show how to find the 2nd or higher leading terms of the late time expressions for $E(t)$ and $\gamma(t)$.

The aim of this paper is to find analytical expressions for the survival amplitude $a(t)$, the effective Hamiltonian $h(t)$ governing the time evolution in the subspace of the unstable states considered and analytical late time 
expressions for $a(t), h(t)$ and also of the instantaneous energy $E(t)$ and decay rate $\gamma(t)$ with the accuracy to the first two non-trivial leading elements of the asymptotic series expansions of these quantities within the model considered.

The paper is organized as follows. Section 2 contains a brief description of general properties of evolving in time unstable states and basic definitions of quantities discussed in next Sections. In Sect. 3 the model of an unstable state defined by the truncated Breit-Wigner energy density distribution function is analyzed: There are found analytical expressions for the survival amplitude $a(t)$, effective Hamiltonian $h(t)$ as well as the late time asymptotic series expansions of $a(t), h(t), E(t)$ and $\gamma(t)$. Section 4 contains graphical presentations of results of numerical calculations of quantities discussed in Section 3. In Sec. 5 one finds a discussion and final remarks.

\section{Preliminaries}

Studying quantum unstable systems one usually analyzes their decay law (that is in their survival probability), which contains a main information about properties of such systems. If one knows that the system is in the initial unstable state $|\phi\rangle \in \mathcal{H},(\mathcal{H}$ is the Hilbert space of states of the considered system), which was prepared at the initial instant $t_{0}=0$, then one can calculate its survival probability, $\mathcal{P}(t)$, of the unstable state $|\phi\rangle$ decaying in vacuum, which equals

$$
\mathcal{P}(t)=|a(t)|^{2},
$$

where $a(t)$ is the probability amplitude of finding the system at the time $t$ in the initial unstable state $|\phi\rangle$,

$$
a(t)=\langle\phi \mid \phi(t)\rangle .
$$

and $|\phi(t)\rangle$ is the solution of the Schrödinger equation for the initial condition $|\phi(0)\rangle=|\phi\rangle$,

$$
i \hbar \frac{\partial}{\partial t}|\phi(t)\rangle=H|\phi(t)\rangle \text {. }
$$

Here $|\phi\rangle,|\phi(t)\rangle \in \mathcal{H}$, and $H$ denotes the total self-adjoint Hamiltonian for the system considered. We assume that there exists a common inertial reference rest frame $\mathcal{O}_{0}$ for the observer and for the unstable system. So, $\mathcal{P}(t)$ is the probability of finding the system at time $t$ in the rest reference frame $\mathcal{O}_{0}$ in the initial unstable state $|\phi\rangle$. 
An important property of the state $|\phi\rangle$ representing an unstable state is that the $|\phi\rangle$ cannot be an eigenvector for $H$ : Simply in such a case the eigenvalue equation $H|\phi\rangle=E_{\phi}|\phi\rangle$ has no solutions.

An unstable state $|\phi\rangle$ can be modeled as a wave packets using solutions of the following eigenvalue equation

$$
H|E\rangle=E|E\rangle, \quad E \in \sigma_{c}(H),
$$

where $\sigma_{c}(H)$ denotes a continuum spectrum of $H$. Eigenvectors $|E\rangle$ are normalized as usual:

$$
\left\langle E \mid E^{\prime}\right\rangle=\delta\left(E-E^{\prime}\right) .
$$

Using vectors $|E\rangle$ we can model an unstable state as the following wavepacket

$$
|\phi\rangle \equiv|\phi\rangle=\int_{E_{\min }}^{\infty} c(E)|E\rangle d E,
$$

where expansion coefficients $c(E)$ are functions of the energy $E$ and $E_{\text {min }}$ is the lower bound of the spectrum $\sigma_{c}(H)$ of $H$. The state $|\phi\rangle$ is normalized $\langle\phi \mid \phi\rangle=1$, which means that it has to be $\int_{E_{\text {min }}}^{\infty}|c(E)|^{2} d E=1$.

Using the definition of the survival amplitude $a(t)$, the expansion (6) and the relation (4) we can find $a(t)$, which takes the following form within the formalism considered,

$$
a(t) \equiv \int_{E_{\min }}^{\infty} \omega(E) e^{-i E t} d E,
$$

where $\omega(E) \equiv|c(E)|^{2}>0$.

As it is seen from (7), the amplitude $a(t)$, and thus the decay law $\mathcal{P}(t)$ of the unstable state $|\phi\rangle$, are completely determined by the density of the energy distribution $\omega(E)$ for the system in this state [14] (see also: [2, 3, 15, 16, 17, 18, 19, 20. Now if to apply Riemann-Lebesque lemma to (7) then the conclusion follows: $a(t) \rightarrow 0$ as $t \rightarrow \infty$. It is because the normalization condition $a(0) \equiv \int_{\text {Spec. }(H)} \omega(E) d m=1$ ensures the absolute integrability of $\omega(E)$. So it has to be $\mathcal{P}(t) \rightarrow 0$ in the case considered. (It appears that this approach can be also applied for Quantum Field Theory models [21, 22, 23]).

Now if to follow Khalfin [2] and to assume that the spectrum of $H$ must be bounded from below, $E_{\text {min }}>-\infty$, and to use the Paley-Wiener Theorem [24] then one comes to the Khalfin's conclusion that in the case of unstable states 
there must be $|a(t)| \geq A \exp \left[-b t^{q}\right]$, for $|t| \rightarrow \infty$. Here $A>0, b>0$ and $0<q<1$. This means that the decay law $\mathcal{P}(t)$ of unstable states decaying in the vacuum, (1), can not be described by an exponential function of time $t$ if time $t$ is suitably long, $t \rightarrow \infty$, and that for these lengths of time $\mathcal{P}(t)$ tends to zero as $t \rightarrow \infty$ more slowly than any exponential function of $t$. Not so long ago this this effect was confirmed by Rothe and his group in experiment described in [5].

It appears that an information about the decay law $\mathcal{P}_{\phi}(t)$ of the state $|\phi\rangle$, strictly speaking about the decay rate $\gamma_{0}$ of this state, as well as the energy $E_{0}$ of the system in this state can be be extracted from $a(t)$. One can do this using the rigorous equation governing the time evolution in the subspace of unstable states, $\mathcal{H}_{\|} \ni|\phi\rangle_{\|} \equiv|\phi\rangle$. Such an equation can be derived using the Schrödinger equation (3) for the total state space $\mathcal{H}$. Namely starting from (3) one finds that within the problem considered.

$$
i \hbar \frac{\partial}{\partial t}\langle\phi \mid \phi(t)\rangle=\langle\phi|H| \phi(t)\rangle \text {. }
$$

So taking into account (2) it can be said that the amplitude $a(t)$ satisfies the following equation

$$
i \hbar \frac{\partial a(t)}{\partial t}=h(t) a(t)
$$

where

$$
h(t)=\frac{\langle\phi|H| \phi(t)\rangle}{a(t)} \equiv \frac{\langle\phi|H| \phi(t)\rangle}{\langle\phi \mid \phi(t)\rangle},
$$

and $h(t)$ is the effective Hamiltonian governing the time evolution in the subspace of unstable states $\mathcal{H}_{\|}=P \mathcal{H}$, where $P$ is the projection operator. The subspace $\mathcal{H}_{\|}$can describe an one-component unstable subsystem and then $P=|\phi\rangle\langle\phi|$ (see [25], [26] and also [12, 13] and references therein) or multi-component subsystem (like neutral kaons complex) and other the like (see: [27, 28, 29] and references one can find therein). The subspace $\mathcal{H} \ominus \mathcal{H}_{\|}=\mathcal{H}_{\perp} \equiv Q \mathcal{H}$ is the subspace of decay products. Here $Q=\mathbb{I}-P$. An equivalent formula for $h(t)$ has the following form [12, 13, 25]:

$$
h(t) \equiv \frac{i \hbar}{a(t)} \frac{\partial a(t)}{\partial t} .
$$

The effective Hamiltonian $h(t)$ is used when one starts with the Schrödinger equation for the total state space $\mathcal{H}$ and looks for the rigorous evolution 
equation for a distinguished subspace of states $\mathcal{H}_{\|} \subset \mathcal{H}$ (see, eg. 25] [30] and also [19, 20]). In general $h(t)$ is a complex function of time. In the case of $\mathcal{H}_{\|}$of dimension two or more the effective Hamiltonian governing the time evolution in such a subspace is a non-hermitian matrix $H_{\|}$or a non-hermitian operator [26, 27, 30]. We have

$$
h(t)=E(t)-\frac{i}{2} \gamma(t)
$$

and

$$
E(t)=\Re[h(t)], \quad \gamma(t)=-2 \Im[h(t)],
$$

are the instantaneous mass energy $E(t)$ and the instantaneous decay rate, $\gamma(t)$ (see [25] and [12, 13]). Here $\Re(z)$ and $\Im(z)$ denote the real and imaginary parts of $z$ respectively. Relations (9), (11) and (13) are very helpful when the density $\omega(E)$ is given and one wants to find the instantaneous energy $E(t)$ and decay rate $\gamma(t)$ : In such a case inserting $\omega(E)$ into (7) one obtains the amplitude $a(t)$ and then using (11) one finds the $h(t)$ and thus $E(t)$ and $\gamma(t)$.

In closing this Section we should pay attention to another problem: The vector $|\phi\rangle$ of the form (6) describing a quantum unstable subsystem can not be an eigenvector of the Hamiltonian $H$, otherwise it would be that $\mathcal{P}(t)=|\langle\phi \mid \phi(t)\rangle|^{2}=\left|\left\langle\phi \mid \exp \left[-\frac{i}{\hbar} t H\right] \phi\right\rangle\right|^{2} \equiv 1$ for all times $t$. The fact that this vector $|\phi\rangle$ is not the eigenvector for $H$ means that the energy of the quantum unstable object is not defined. Simply the energy can not take the exact constant value in this state $|\phi\rangle$. In such a case quantum systems are characterized by the energy distribution density $\omega(E)$ and the average energy

$<E>=\int_{E_{\min }}^{\infty} E \omega(E) d E$ or by the instantaneous energy $E(t)$ but not by the exact value of the energy.

\section{A unstable system defined by the truncated Breit-Wigner energy density distribution: analytical results}

In the large literature many quantum unstable systems are described within the Fock-Krylov theory using Breit-Wigner energy density distribution function $\omega_{B W}(E)$. The use of $\omega_{B W}(E)$ is convenient because it describes relatively well a large class of unstable systems and allows to find analytical form of the 
survival amplitude $a(t)$ (see, eg. [11, 12, 13] and other papers). It appears that for this energy density distribution one can find also analytical form of $a(t)$ at very late times as well as analytical asymptotic form of $h(t), E(t)$ and $\gamma(t)$ for such times.

\subsection{A survival amplitude}

Let us assume that $\operatorname{Spec} .(H)=\left[E_{\text {min }}, \infty\right)$ and let us choose $\omega(E)$ as follows

$$
\omega(E) \equiv \omega_{B W}(E) \stackrel{\text { def }}{=} \frac{N}{2 \pi} \Theta\left(E-E_{\text {min }}\right) \frac{\gamma_{0}}{\left(E-E_{0}\right)^{2}+\left(\frac{\gamma_{0}}{2}\right)^{2}},
$$

where $N$ is a normalization constant and $\Theta(E)$ is the unit step function: $\Theta(E)=1$ for $E \geq 0$ and $\Theta(E)=0$ for $E<0$. For such $\omega(E)$ using the integral representation of the survival amplitude (7) one finds

$$
a(t)=\frac{N}{2 \pi} \int_{E_{\min }}^{\infty} \frac{\gamma_{0}}{\left(E-E_{0}\right)^{2}+\left(\frac{\gamma_{0}}{2}\right)^{2}} e^{-\frac{i}{\hbar} E t} d E,
$$

where

$$
\frac{1}{N}=\frac{1}{2 \pi} \int_{E_{\text {min }}}^{\infty} \frac{\gamma_{0}}{\left(E-E_{0}\right)^{2}+\left(\frac{\gamma_{0}}{2}\right)^{2}} d E .
$$

Using dimensionless variables

$$
\eta=\frac{E-E_{\min }}{\gamma_{0}}, \quad \beta=\frac{E_{0}-E_{\min }}{\gamma_{0}} \text { and } \tau=\frac{\gamma_{0} t}{\hbar} \stackrel{\text { def }}{=} \tau(t),
$$

the integral (15) can be rewritten in the following form

$$
\begin{aligned}
a(t) \equiv a(\tau(t)) & =\frac{N}{2 \pi} e^{-\frac{i}{\hbar} E_{\min } t} e^{-i \beta \tau} \int_{-\beta}^{\infty} \frac{1}{\eta^{2}+\frac{1}{4}} e^{-i \eta \tau} d \eta \\
& =\frac{N}{2 \pi} e^{-\frac{i}{\hbar} E_{\min } t} e^{-i \beta \tau} \times I_{\beta}(\tau)
\end{aligned}
$$

where

$$
I_{\beta}(\tau) \stackrel{\text { def }}{=} \int_{-\beta}^{\infty} \frac{1}{\eta^{2}+\frac{1}{4}} e^{-i \eta \tau} d \eta .
$$

After some algebra one can express the function $I_{\beta}(t)$ defined by the relation (20) in terms of the integral-exponential functions $E_{1}(z)$,

$$
\begin{gathered}
I_{\beta}(t)=I_{\beta}(\tau(t)) \equiv 2 \pi e^{-\frac{\tau}{2}\left\{1-\frac{i}{2 \pi}\left[e^{\tau} E_{1}\left(-i\left(\beta+\frac{i}{2}\right) \tau\right)+\right.\right.} \\
\left.\left.+(-1) E_{1}\left(-i\left(\beta-\frac{i}{2}\right) \tau\right)\right]\right\}
\end{gathered}
$$


wherein $E_{1}(z)$ is defined according to formula 6.2.1 in [31. From (21) and (19) the following formula follows

$$
\begin{aligned}
a(t) \equiv a(\tau(t))= & N e^{-i\left(\beta-\frac{i}{2}\right) \tau} \times \\
\times & \left\{e^{-\frac{i}{\hbar} E_{\text {min }} t}-\frac{i}{2 \pi}\left[e^{\tau} E_{1}\left(-i\left(\beta+\frac{i}{2}\right) \tau\right)\right.\right. \\
& \left.\left.+(-1) E_{1}\left(-i\left(\beta-\frac{i}{2}\right) \tau\right)\right]\right\}
\end{aligned}
$$

or equivalently,

$$
\begin{aligned}
a(t)= & N e^{-\frac{i}{\hbar}\left(E_{0}-i \frac{\gamma_{0}}{2}\right) t} \times \\
\times & \left\{1-\frac{i}{2 \pi}\left[e^{\frac{\gamma_{0} t}{\hbar}} E_{1}\left(-\frac{i}{\hbar}\left(E_{R}+\frac{i}{2} \gamma_{0}\right) t\right)+\right.\right. \\
& \left.\left.+(-1) E_{1}\left(-\frac{i}{\hbar}\left(E_{R}-\frac{i}{2} \gamma_{0}\right) t\right)\right]\right\}
\end{aligned}
$$

where $E_{R}=E_{0}-E_{\min }$.

The results (22) and (23) mean in general $|a(t)|^{2}$ is not a pure exponential function of time that within the model considered. What is more it appears that the survival amplitude $a(t)$ can not coincide with the canonical survival amplitude $a_{c}(t)$,

$$
a_{c}(t) \stackrel{\text { def }}{=} A e^{-\frac{i}{\hbar}\left(E_{0}-\frac{i}{2} \gamma_{0}\right) t},
$$

at any finite time interval. In order to see that let us assume that there exist such a time interval $\left[t_{1}, t_{2}\right]$, where $t_{1}<t_{2}$, that

$$
a(t)=a_{c}(t),
$$

for all $t \in\left[t_{1}, t_{2}\right]$. Then using (23) one finds that it should be in such a case:

$$
\begin{aligned}
A e^{-\frac{i}{\hbar}\left(E_{0}-\frac{i}{2} \gamma_{0}\right) t \equiv} & N e^{-\frac{i}{\hbar}\left(E_{0}-i \frac{\gamma_{0}}{2}\right) t} \times \\
\times & \left\{1-\frac{i}{2 \pi}\left[e^{\frac{\gamma_{0} t}{\hbar}} E_{1}\left(-\frac{i}{\hbar}\left(E_{R}+\frac{i}{2} \gamma_{0}\right) t\right)\right.\right. \\
& \left.\left.+(-1) E_{1}\left(-\frac{i}{\hbar}\left(E_{R}-\frac{i}{2} \gamma_{0}\right) t\right)\right]\right\},
\end{aligned}
$$

This means that

$$
\begin{aligned}
1-\frac{A}{N}=\frac{i}{2 \pi} & {\left[e^{\frac{\gamma_{0} t}{\hbar}} E_{1}\left(-\frac{i}{\hbar}\left(E_{R}+\frac{i}{2} \gamma_{0}\right) t\right)\right.} \\
& \left.+(-1) E_{1}\left(-\frac{i}{\hbar}\left(E_{R}-\frac{i}{2} \gamma_{0}\right) t\right)\right],
\end{aligned}
$$


or,

$$
\begin{aligned}
\chi(t) \stackrel{\text { def }}{=} e^{\frac{\gamma_{0} t}{\hbar}} E_{1}\left(-\frac{i}{\hbar}\left(E_{R}+\frac{i}{2} \gamma_{0}\right) t\right) & \\
& -E_{1}\left(-\frac{i}{\hbar}\left(E_{R}-\frac{i}{2} \gamma_{0}\right) t\right)=c \equiv \text { const. }
\end{aligned}
$$

for $t \in\left[t_{1}, t_{2}\right]$. So, there should be in the considered case,

$$
\frac{d \chi(t)}{d t}=0
$$

for $t \in\left[t_{1}, t_{2}\right]$. Using (26) one finds that

$$
\frac{d \chi(t)}{d t} \equiv \frac{\gamma_{0}}{\hbar} e^{\frac{\gamma_{0} t}{\hbar}} E_{1}\left(-\frac{i}{\hbar}\left(E_{R}+\frac{i}{2} \gamma_{0}\right) t\right)
$$

From the last relation and from properties of integral-exponential function $E_{1}(z)$ one concludes that the condition $\frac{\partial \chi(t)}{d t}=0$ can be satisfied for isolated values of time $t$ at most. This means that there does not exist any time interval $\left[t_{1}, t_{2}\right]$ such that $\chi(t)=$ const for all $t \in\left[t_{1}, t_{2}\right]$, or that there does not exist any time interval $\left[t_{1}, t_{2}\right]$ and any $A \neq 0$ such that for $t \in\left[t_{1}, t_{2}\right]$ it could be $\mathcal{P}(t) \equiv|a(t)|^{2}=\left|a_{c}(t)\right|^{2} \equiv|A|^{2} \exp \left[-\gamma_{0} t\right]$ in the considered model defined by $\omega(E)=\omega_{B W}(E)$.

\subsection{Instantaneous energy $E(t)$ and decay rate $\gamma(t)$}

Not let us analyze properties of the instantaneous energy $E(t)$ and instantaneous decay rate $\gamma(t)$ in the model considered. These quantities are defined using the effective Hamiltonian $h(t)$. In order to find $h(t)$ we need for the quantity $i \hbar \frac{\partial a(t)}{\partial t}($ see (11) $)$. From (19) one finds that

$$
i \hbar \frac{\partial a(t)}{\partial t}=E_{0} a(t)+\gamma_{0} \frac{N}{2 \pi} e^{-\frac{i}{\hbar} E_{0} t} J_{\beta}(\tau(t))
$$

where

$$
J_{\beta}(\tau)=\int_{-\beta}^{\infty} \frac{x}{x^{2}+\frac{1}{4}} e^{-i x \tau} d x
$$

or simply (see (200)

$$
J_{\beta}(\tau) \equiv i \frac{\partial I_{\beta}(\tau)}{\partial \tau}
$$


This last relation is very convenient when one tries to find an analytical expression for $J_{\beta}(\tau)$ : One just has to find an analytical formula for $I_{\beta}(\tau)$ and then use relation (30).

Now the use (19), (28) and (11) leads to the conclusion that

$$
h(t)=i \hbar \frac{1}{a(t)} \frac{\partial a(t)}{\partial t}=E_{0}+\gamma_{0} \frac{J_{\beta}(\tau(t))}{I_{\beta}(\tau(t))},
$$

which means that

$$
E(t)=\Re[h(t)]=E_{0}+\gamma_{0} \Re\left[\frac{J_{\beta}(\tau(t))}{I_{\beta}(\tau(t))}\right],
$$

and

$$
\gamma(t)=-2 \Im[h(t)]=-2 \gamma_{0} \Im\left[\frac{J_{\beta}(\tau(t))}{I_{\beta}(\tau(t))}\right] .
$$

In order to visualize properties of $E(t)$ it is convenient to use the following function:

$$
\kappa(t) \stackrel{\text { def }}{=} \frac{E(t)-E_{\min }}{E_{0}-E_{\min }} .
$$

Using (32) one finds that

$$
E(t)-E_{\text {min }}=E_{0}-E_{\text {min }}+\gamma_{0} \Re\left[\frac{J_{\beta}(\tau)}{I_{\beta}(\tau)}\right],
$$

If to divide two sides of the above equation by $E_{0}-E_{\min }$ then one obtains the function $\kappa(t)$ (see (34)) we are looking for:

$$
\kappa(\tau(t))=1+\frac{1}{\beta} \Re\left[\frac{J_{\beta}(\tau(t))}{I_{\beta}(\tau(t))}\right] .
$$

An alternative analytical formula for $h(t)$ can be obtained using analytical expressions for $a(t)$ and $\frac{\partial a(t)}{\partial t}$. One can express the integral (29) defining $J_{\beta}(\tau)$ in terms of the exponential integral functions, which allows us to rewrite the formula (28) for $i \hbar \frac{\partial a(t)}{\partial t}$ as follows

$$
\begin{aligned}
i \hbar \frac{\partial a(t)}{\partial t}= & E_{0} a(t)+(-i) \gamma_{0} \frac{N}{2} e^{-\frac{i}{\hbar}\left(E_{0}-\frac{i}{2} \gamma_{0}\right) t} \times \\
& \times\left\{1+\frac{i}{2 \pi}\left[e^{\tau} E_{1}\left[-i\left(\beta+\frac{i}{2}\right) \tau\right]+E_{1}\left[-i\left(\beta-\frac{i}{2}\right) \tau\right]\right]\right\} .
\end{aligned}
$$


This relation together with (22) gives

$$
\begin{aligned}
h(t) \equiv & E_{0}-\frac{i}{2} \gamma_{0} \times \\
& \times \frac{1+\frac{i}{2 \pi}\left[e^{\tau} E_{1}\left[-i\left(\beta+\frac{i}{2}\right) \tau\right]+E_{1}\left[-i\left(\beta-\frac{i}{2}\right) \tau\right]\right]}{1-\frac{i}{2 \pi}\left[e^{\tau} E_{1}\left(-i\left(\beta+\frac{i}{2}\right) \tau\right)-E_{1}\left(-i\left(\beta-\frac{i}{2}\right) \tau\right)\right]} .
\end{aligned}
$$

Taking the real part of $h(t)$ given by the last relation one obtains $E(t) \equiv$ $\Re[h(t)]$ and then one can calculate $\kappa(t)$ and so on.

\subsection{Late time properties of $a(t), h(t)$ and $\gamma(t)$}

The late time asymptotic form of $a(t)$ and $h(t)$ can be found using e.g. the formulae (20), (31) respectively. In order to do this for the beginning we should find the asymptotic form of integrals $I_{\beta}(\tau)$ and $J_{\beta}(\tau)$ used in the formula (31) for $h(t)$. These integrals are defined by the expressions (20) and (29) respectively. It is relatively simple to find asymptotic expressions

$I_{\beta}(\tau)$ and $J_{\beta}(\tau)$ for $\tau \rightarrow \infty$ directly from (20) and (29) using, e.g., the method of the integration by parts. We have for $\tau \rightarrow \infty$

$$
\begin{gathered}
I_{\beta}(\tau) \simeq \frac{i}{\tau} \frac{e^{i \beta \tau}}{\beta^{2}+\frac{1}{4}\{-1}+\frac{2 \beta}{\beta^{2}+\frac{1}{4}} \frac{i}{\tau}+\frac{2}{\beta^{2}+\frac{1}{4}}\left[1-\frac{4 \beta^{2}}{\beta^{2}+\frac{1}{4}}\right]\left(\frac{i}{\tau}\right)^{2} \\
+\frac{24 \beta}{\left(\beta^{2}+\frac{1}{4}\right)^{2}}\left[\frac{2 \beta^{2}}{\beta^{2}+\frac{1}{4}}-1\right]\left(\frac{i}{\tau}\right)^{3} \\
\left.+\frac{24}{\left(\beta^{2}+\frac{1}{4}\right)^{2}}\left[-\frac{16 \beta^{4}}{\left(\beta^{2}+\frac{1}{4}\right)^{2}}+\frac{12 \beta^{2}}{\beta^{2}+\frac{1}{4}}-1\right]\left(\frac{i}{\tau}\right)^{4}+\ldots\right\},
\end{gathered}
$$

and

$$
\begin{aligned}
& J_{\beta}(\tau) \simeq \frac{i}{\tau} \frac{e^{i \beta \tau}}{\beta^{2}+\frac{1}{4}}\left\{\beta+\left[1-\frac{2 \beta^{2}}{\beta^{2}+\frac{1}{4}}\right] \frac{i}{\tau}+\frac{2 \beta}{\beta^{2}+\frac{1}{4}}\left[\frac{4 \beta^{2}}{\beta^{2}+\frac{1}{4}}-3\right]\left(\frac{i}{\tau}\right)^{2}\right. \\
& +\frac{6}{\beta^{2}+\frac{1}{4}}\left[-\frac{8 \beta^{4}}{\left(\beta^{2}+\frac{1}{4}\right)^{2}}+\frac{8 \beta^{2}}{\beta^{2}+\frac{1}{4}}-1\right]\left(\frac{i}{\tau}\right)^{3} \\
& \left.+\frac{24 \beta}{\left(\beta^{2}+\frac{1}{4}\right)^{2}}\left[\frac{16 \beta^{4}}{\left(\beta^{2}+\frac{1}{4}\right)^{2}}-\frac{20 \beta^{2}}{\beta^{2}+\frac{1}{4}}+5\right]\left(\frac{i}{\tau}\right)^{4} \ldots\right\} \text {. }
\end{aligned}
$$


These two last asymptotic expressions allow one to find for $\tau \rightarrow \infty$ the asymptotic form of the ratio $\frac{J_{\beta}(\tau)}{I_{\beta}(\tau)}$ used in relations (31), (32) and (35) $)$, which has much simpler form than asymptotic expansions for $I_{\beta}(\tau)$ and $J_{\beta}(\tau)$. In order to do this let us define an auxiliary function $\phi(x)$,

$$
\frac{J_{\beta}(\tau)}{I_{\beta}(\tau)} \equiv \phi(x)=\frac{\beta+a_{1} x+a_{2} x^{2}+a_{3} x^{3}+a_{4} x^{4}+\ldots}{-1+b_{1} x+b_{2} x^{2}+b_{3} x^{3}+b_{4} x^{4} \ldots} .
$$

where $x=i / \tau$. Now taking into account that at late times $1 / \tau \ll 1$, which means that $|x| \ll 1$ at this times region and expanding $\phi(x)$ given by (41) in Taylor series around $x=0$ one finds after some algebra that

$$
\begin{aligned}
\phi(x) \simeq & -\beta-x+\frac{2 \beta}{\beta^{2}+\frac{1}{4}} x^{2}+ \\
& +\frac{1}{4} \frac{1+24 \beta-28 \beta^{2}-96 \beta^{3}+64 \beta^{4}}{\left(\beta^{2}+\frac{1}{4}\right)^{3}} x^{3} \\
& +\frac{1}{4} \frac{6-21 \beta+48 \beta^{2}-64 \beta^{3}-288 \beta^{4}+464 \beta^{5}}{\left(\beta^{2}+\frac{1}{4}\right)^{4}} x^{4}+\ldots,
\end{aligned}
$$

for $|x| \ll 1$. Hence, there is for $\tau \rightarrow \infty$,

$$
\begin{aligned}
\frac{J_{\beta}(\tau)}{I_{\beta}(\tau)} \simeq & -\beta-\frac{i}{\tau}-\frac{2 \beta}{\beta^{2}+\frac{1}{4}} \frac{1}{\tau^{2}} \\
& -i \frac{1}{4} \frac{1+24 \beta-28 \beta^{2}-96 \beta^{3}+64 \beta^{4}}{\left(\beta^{2}+\frac{1}{4}\right)^{3}} \frac{1}{\tau^{3}} \\
& +\frac{1}{4} \frac{6-21 \beta+48 \beta^{2}-64 \beta^{3}-288 \beta^{4}+464 \beta^{5}}{\left(\beta^{2}+\frac{1}{4}\right)^{4}} \frac{1}{\tau^{4}}+\ldots,
\end{aligned}
$$

where $\tau$ and $\beta$ are defined by formulae (17) respectively.

Using relation (19) one finds from (39) the late time asymptotic form 
$a_{l t}(t)$ of the survival amplitude $a(t)$. There is

$$
\begin{aligned}
a_{l t}(t) \stackrel{\text { def }}{=} & \left.a(t)\right|_{t \rightarrow \infty}=\frac{N}{2 \pi} \frac{e^{-\frac{i}{\hbar} E_{\text {min }} t}}{\beta^{2}+\frac{1}{4}} \times \\
& \times\left\{-i \frac{\hbar}{\gamma_{0} t}-\frac{2 \beta}{\beta^{2}+\frac{1}{4}}\left(\frac{\hbar}{\gamma_{0} t}\right)^{2}\right. \\
& -i \frac{2}{\beta^{2}+\frac{1}{4}}\left[1-\frac{4 \beta^{2}}{\beta^{2}+\frac{1}{4}}\right]\left(\frac{\hbar}{\gamma_{0} t}\right)^{3} \\
& \left.+\frac{24 \beta}{\left(\beta^{2}+\frac{1}{4}\right)^{2}}\left[\frac{2 \beta^{2}}{\beta^{2}+\frac{1}{4}}-1\right]\left(\frac{\hbar}{\gamma_{0} t}\right)^{4}+\ldots\right\} .
\end{aligned}
$$

Starting from the asymptotic expression (43) and using formula (31) one can find the late time asymptotic form of $h(t)$ and thus of $E(t)$ and $\gamma(t)$ for model considered,

$$
\begin{aligned}
\left.E(t)\right|_{t \rightarrow \infty}= & \Re\left[h(t) \|_{t \rightarrow \infty}\right. \\
\simeq & E_{\min }-2 \frac{E_{0}-E_{\min }}{\gamma_{0}^{2}\left(\beta^{2}+\frac{1}{4}\right)}\left(\frac{\hbar}{t}\right)^{2} \\
& +\frac{1}{4} \frac{6-21 \beta+48 \beta^{2}-64 \beta^{3}-288 \beta^{4}+464 \beta^{5}}{\gamma_{0}^{3}\left(\beta^{2}+\frac{1}{4}\right)^{4}}\left(\frac{\hbar}{t}\right)^{4}+\ldots,
\end{aligned}
$$

and,

$$
\begin{aligned}
\left.\gamma(t)\right|_{t \rightarrow \infty} & =-2 \Im[h(t)] \\
& \simeq 2 \frac{\hbar}{t}+\frac{1}{2} \frac{1+24 \beta-28 \beta^{2}-96 \beta^{3}+64 \beta^{4}}{\gamma_{0}^{2}\left(\beta^{2}+\frac{1}{4}\right)^{3}}\left(\frac{\hbar}{t}\right)^{3}+\ldots
\end{aligned}
$$

These three last relations are valid for $t>T$, where $T$ denotes the cross-over time, i.e. the time when canonical exponential and late time inverse power law contributions to the survival amplitude become comparable:

$$
\left|a_{c}(t)\right|^{2} \simeq\left|a_{l t}(t)\right|^{2}
$$

The cross-over time $T$ is the solution of the last equation.

\section{Numerical results}

This Section contains results of numerical studies of the the quantities analyzed in the previous Section. Their results were obtained for the chosen 
values of $\beta$ and are presented graphically in Figs (11) - (4). In all figures one can find a typical form of the quantities characterizing properties of the unstable state as a function of time: Decay curves $\mathcal{P}(t)$, instantaneous decay rates $\gamma(t)$ and instantaneous energies $E(t)$.
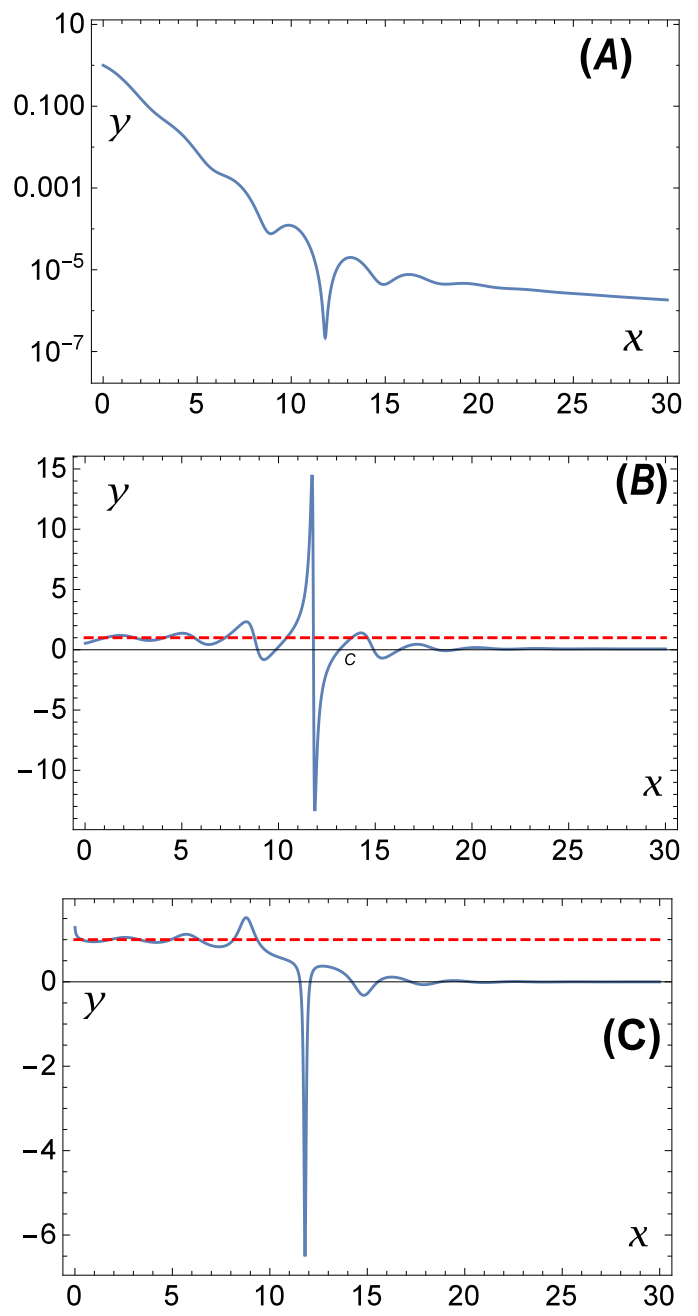

Figure 1: $(A)$ The decay curve, $(B)$ The instantaneous decay rate, $(C)$ The instantaneous energy. Axes: $x$ - in all panels time $t$ measured in lifetimes $\tau$ : $x=t / \tau ;(A)-y=\mathcal{P}(t / \tau)$, (The logarithmic scale); $(B)-y=\gamma(t / \tau) / \gamma_{0} ;$ $(C)-\kappa(t / \tau),(\kappa(t / \tau)$ is defined by formula (34) $)$. The case $\beta=2$. 



Figure 2: $(A)$ The decay curve, $(B)$ The instantaneous decay rate, $(C)$ The instantaneous energy. Axes: $x$ - in all panels time $t$ measured in lifetimes $\tau$ : $x=t / \tau ;(A)-y=\mathcal{P}(t / \tau),\left(\right.$ The logarithmic scale); $(B)-y=\gamma(t / \tau) / \gamma_{0} ;$ $(C)-\kappa(t / \tau),(\kappa(t / \tau)$ is defined by formula (34) $)$. The case $\beta=10$. 

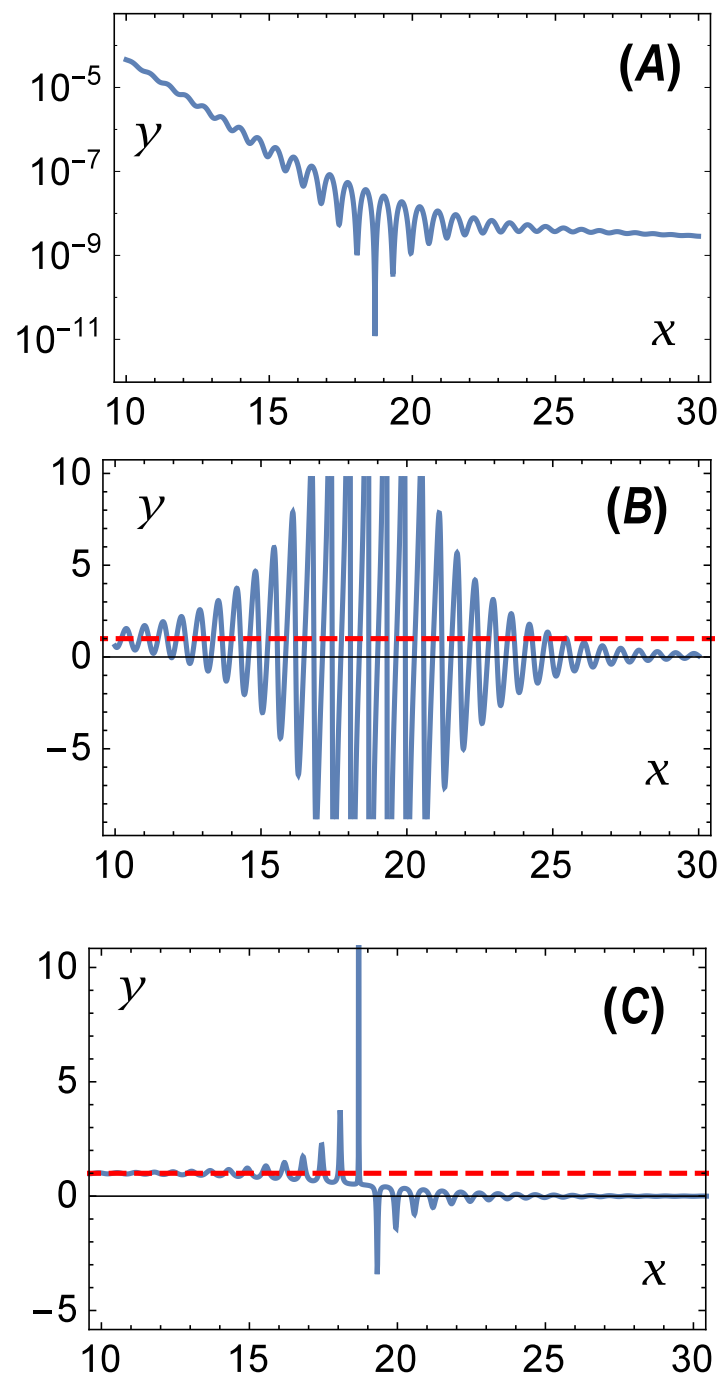

Figure 3: The same as in the Fig (2): The transition times region: $t \sim T$. 

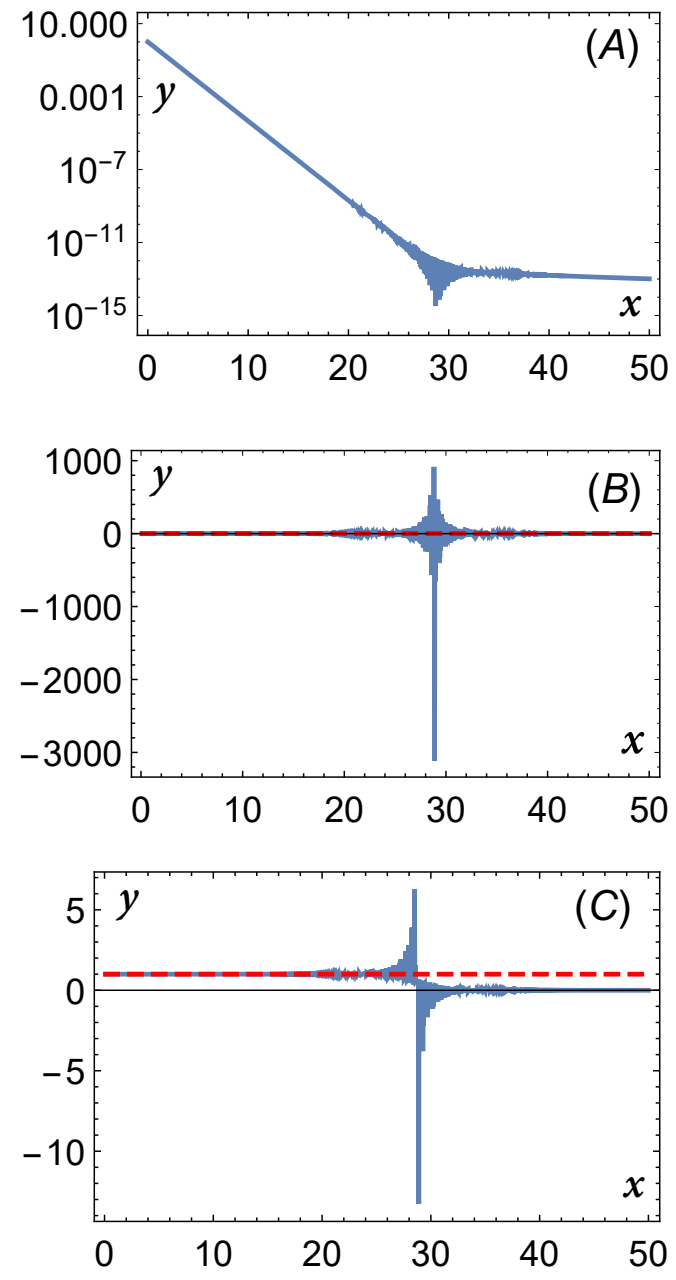

Figure 4: $(A)$ The decay curve, $(B)$ The instantaneous decay rate, $(C)$ The instantaneous energy. Axes: $x$ - in all panels time $t$ measured in lifetimes $\tau$ : $x=t / \tau ;(A)-y=\mathcal{P}(t / \tau)$, (The logarithmic scale); $(B)-y=\gamma(t / \tau) / \gamma_{0} ;$ $(C)-\kappa(t / \tau),(\kappa(t / \tau)$ is defined by formula (34) $)$. The case $\beta=100$.

In all Figures: The horizontal dashed line in panels $(B)$ denotes the situation when $\frac{\gamma(t / \tau)}{\gamma_{0}}=1$ (that is when $\gamma(t / \tau)=\gamma_{0}$ ) and the horizontal dashed line in panels $(C)$ denotes the case $\kappa(t / \tau)=1$ which is equivalent to the condition $E(t / \tau)=E_{0}$. 


\section{Discussion and final remarks}

A similar form of a decay curves to those presented in panels $(A)$ of Figs (1) - (4) one meets for a very large class of models defined by energy densities $\omega(E)$ of the following type

$$
\begin{aligned}
\omega(E)=\frac{N}{2 \pi} & \Theta\left(E-E_{\text {min }}\right)\left(E-E_{\text {min }}\right)^{\lambda} \times \\
& \times \frac{\gamma_{\phi}^{0}}{\left(E-E_{0}\right)^{2}+\frac{\left(\gamma_{0}\right)^{2}}{4}} f(E),
\end{aligned}
$$

where $\lambda \geq 0, f(E)$ is a form-factor - it is a smooth function going to zero as $E \rightarrow \infty$ and it has no threshold and no pole. The asymptotical large time behavior of $a(t)$ is due to the term $\left(E-E_{\text {min }}\right)^{\lambda}$ and the choice of $\lambda$. The density $\omega(E)$ defined by the relation (48) fulfills all physical requirements and it leads to the decay curves having a very similar form at transition times region (where $t \sim T$ ) to the decay curves presented above. The characteristic feature of all these decay curves is the presence of sharp and frequent oscillations at the transition times region. This means that derivatives of the amplitude $a(t)$ may reach extremely large values for some times from this time region and the modulus of these derivatives is much larger than the modulus of $a(t)$, which is very small for these times. This explains why in this time region the real and imaginary parts of $h(t) \equiv E(t)-\frac{i}{2} \gamma(t)$, which can be expressed by the relation (11), ie. by a large derivative of $a(t)$ divided by a very small $a(t)$, reach values much larger than the energy $E_{0}$ of the the unstable state measured at times for which the decay curve has the exponential form.

Results presented in Sec. 3 shows that within the model considered there does not exist a finite time interval where it could be $a(t)=a_{c}(t)$. So one can expect $\left|a(t)-a_{c}(t)\right| \neq 0$ and varies over time $t$ not only at times $t \sim T$ but also even for times $t \ll T$ (see also [32]). On the other hand results of numerical calculations presented in graphical form in Sec. 4 shows that at these times $|a(t)|^{2} \simeq\left|a_{c}(t)\right|^{2}$ to a very good approximation. So the amplitude of the mentioned variations of $\left|a(t)-a_{c}(t)\right|$ in time for $t \ll T$ is rather almost

negligible small. Such a conclusion agrees with the conclusion resulting from analysis of the form of $E(t)$ and $\gamma(t)$ obtained numerically and presented in Sec. 4: We observe that at times $t \ll T E(t) \simeq E_{0}$ and $\gamma(t) \simeq \gamma_{0}$.

Note that the equivalent formula (10) for $h(t)$ means that $h(t)$ is the socalled "weak value". The behavior of $E(t)$ and $\gamma(t)$ at the transition times 
region, where $t \sim T$, presented in Figs (1) - (4) is quite obvious for the weak values.

The question is whether and where this effect can manifest itself. As it was mentioned earlier, the effect presented in panels $(A)$ in Figs (1) - (4), that is the transition of the exponential form of the decay law $\mathcal{P}_{\phi}(t)=|a(t)|^{2}$ into the inverse power law form, was confirmed experimentally by Rhote nad his group. This means that effects presented in panels $(B)$ and $(C)$ of these Figures and resulting from the properties of the amplitude $a(t)$ have to take place for $h(t)$ too, and thus for $E(t)$ and $\gamma(t)$.

It seems that the following cases are the most likely ones where the above described long time properties of unstable states may manifest itself or where they can be observed; (i) One should analyze properties of unstable states having not too long values of the cross-over time $T$, or (ii) one should find a possibility to observe a suitably large number of events, i.e. unstable particles, created by the same source.

The problem of broad resonances in the scalar sector ( $\sigma$ meson problem) discussed in [33, 34], where the hypothesis was formulated that this problem could be connected with properties of the decay amplitude in the transition time region, seems to be possible manifestations of this effect and this problem refers to the first possibility mentioned above.

Astrophysical and cosmological processes in which extremely huge numbers of unstable particles are created seem to be another possibility for the above discussed effect to become manifest. The probability $\mathcal{P}_{\phi}(t)=|a(t)|^{2}$ that an unstable particle, say $\phi$, survives up to time $t \sim T$ is extremely small: Let $\mathcal{P}_{\phi}(t)$ be such that

$$
\left.\mathcal{P}_{\phi}(t)\right|_{t \sim T} \sim 10^{-k}
$$

where $k \gg 1$, then there is a chance to observe some of particles $\phi$ survived at $t \sim T$ only if there is a source creating these particles in $\mathcal{N}_{\phi}$ number such that

$$
\left.\mathcal{P}_{\phi}(t)\right|_{t \sim T} \mathcal{N}_{\phi} \gg 1
$$

So if a source exists that creates a flux containing

$$
\mathcal{N}_{\phi} \sim 10^{l}
$$

unstable particles and $l \gg k$ then the probability theory states that the number $N_{\text {surv }}$ unstable particles

$$
N_{\text {surv }}=\left.\mathcal{P}_{\phi}(t)\right|_{t \sim T} \mathcal{N}_{\phi} \sim 10^{l-k} \gg 0,
$$


has to survive up to time $t \sim T$.

Sources creating such numbers of unstable particles are known from cosmology and astrophysics: The Big Bang; Processes taking place in galactic nuclei (galactic cores); Processes taking place inside stars (supernova explosions); Etc.. So one should look for a manifestation of the quantum effect presented in panels $(B)$ and $(C)$ in Figs (2) - (4) in astronomical observations and in cosmology.

The third case, where late time properties of the instantaneous energy $E(t)$ of the unstable state can can play important role is cosmology. It seems also that the long time properties of the energy $E(t)$ of unstable states can justify the use time dependent cosmological constant $\Lambda$ within the so-called $\Lambda$ Cold Dark Matter $(\Lambda \mathrm{CDM})$ Cosmology. Namely form the literature it is known that the cosmological constant $\Lambda$ of the form,

$$
\Lambda \equiv \Lambda(t) \simeq \Lambda_{\text {bare }}+\frac{D}{t^{2}}
$$

(where $D=$ const.) was considered in many papers: Similar form of $\Lambda$ was obtained in [35, where the invariance under scale transformations of the generalized Einstein equations was studied. Such a time dependence of $\Lambda$ was postulated also in [36] as the result of the analysis of the large numbers hypothesis. The cosmological model with time dependent $\Lambda$ of the above postulated form was studied also in [37]. This form of $\Lambda$ was assumed in eg. in [38] but there was no any explanation what physics suggests such the choice. Cosmological model with time dependent $\Lambda$ were also studied in much more recent papers.

Krauss and Dent in their paper [39, 40] made a hypothesis that some false vacuum regions do survive well up to the time $T$ or later. So, let $|\phi\rangle=|0\rangle^{\text {false }}$, be a false, $|0\rangle^{\text {bare }}$ - a bare, true vacuum states and $E_{0} \equiv E_{0}^{f a l s e}$ be the energy of a state corresponding to the false vacuum measured at the canonical decay time and $E_{0}^{\text {bare }} \equiv E_{\text {min }}$ be the energy of true vacuum (i.e. the true ground state of the system). As it is seen from the results presented in Section 3, the problem is that the energy of those false vacuum regions which survived up to $T$ and much later differs from $E_{0}^{\text {false }}$. Going from quantum mechanics to quantum field theory one should take into account among others a volume factors so that survival probabilities per unit volume per unit time should be considered. The standard false vacuum decay calculations shows that the same volume factors should appear in both early and late time decay rate estimations (see Krauss and Dent [39]). This means that the calculations of 
cross-over time $T$ can be applied to survival probabilities per unit volume. For the same reasons within the quantum field theory the quantity $E(t)$ can be replaced by the energy per unit volume $\rho(t)=E(t) / V$ because these volume factors $V$ appear in the numerator and denominator of the formula (11) for $h(t)$. Therefore assuming that we live in the Unverse with a false vacuum and based on the result (45) one concludes that there should be at times $t>T$ :

$$
E^{\text {false }}(t) \simeq E_{\text {bare }}^{0}+\frac{c_{2}}{t^{2}}+\frac{c_{4}}{t^{4}} \ldots, \text { for } t \gg T,
$$

(where $c_{2}=c_{2}^{*}$ and $c_{4}=c_{4}^{*}$ ), or,

$$
\rho_{0}(t) \stackrel{\text { def }}{=} \rho^{\text {false }}(t) \simeq \rho_{0}^{\text {bare }}+\frac{d_{2}}{t^{2}}+\frac{d_{4}}{t^{4}} \ldots, \text { for } t \gg T,
$$

(where $\rho^{\text {false }}(t)$ is the energy density in the false vacuum state, $d_{2}=d_{2}^{*}, d_{4}=$ $\left.d_{4}^{*}, \rho_{0}(t)=\rho^{\text {false }}(t)=E^{\text {false }}(t) / V, \rho_{0}^{\text {bare }}=E_{0}^{\text {bare }} / V\right)$. The standard relation is

$$
\rho_{0} \equiv \rho_{0}^{\text {bare }}=c^{2} \frac{\Lambda_{0}}{8 \pi G},
$$

where $\Lambda_{0} \equiv \Lambda^{\text {bare }}$ is the bare cosmological constant.

So the relations

$$
\rho_{0}(t)=\rho_{0}^{\text {bare }}+\frac{d_{2}}{t^{2}}+\frac{d_{4}}{t^{4}}, \quad \text { and } \quad \Lambda(t)=\Lambda^{\text {bare }}+\frac{D_{2}}{t^{2}}+\frac{D_{4}}{t^{4}},
$$

(where $D_{2}, D_{4}$ are real) are equivalent and they both are a manifestation of quantum long time properties of unstable states. These last two relations explain why it is reasonable to use $\Lambda$ of the form (53), especially when one considers cosmologies with the false vacua.

Summing up: Late time properties of evolving in time quantum unstable systems are extremely difficult to observe. Discussion presented in this Section shows where there is a chance to observe a manifestation of these late time effects and in which cases taking into account these properties may explain why some theories are worth to a deeper analysis: A good example is the cosmology with time dependent cosmological "constant" $\Lambda(t)$.

\section{References}

[1] V. F. Weisskopf, E. T. Wigner, Z. Phys., 63, 54, (1930); 65, 18, (1930). 
[2] L. A. Khalfin, Zh. Eksp. Teor. Fiz., 33, p.p. 1371 - 1382, (1957) [in Russian]; [Sov. Phys. - JETP , 6, 1053 (1958)].

[3] L. Fonda, G. C. Ghirardii and A. Rimini, Rep. on Prog. in Phys., 41, 587 (1978).

[4] E. B. Norman, et al., Phys. Rev. Lett., 60, 2246 (1988).

[5] C. Rothe, S. I. Hintschich and A. P. Monkman, Phys. Rev. Lett., 96, 163601 (2006).

[6] J. Seke, W. N. Herfort, Phys. Rev., A 38, 833 (1998).

[7] R. E. Parrot, J. Lawrence, Europhys. Lett., 57, 632 (2002).

[8] J. Lawrence, Journ. Opt. B: Quant. Semiclass. Opt., 4, S446 (2002).

[9] I. Joichi, Sh. Matsumoto, M. Yoshimura, Phys. Rev., D 58, 045004 (1998).

[10] T. Jiitoh, S. Matsumoto, J. Sato, Y. Sato, K. Takeda, Phys Rev., A 71, 012109 (2005).

[11] K. M. Sluis, E. A. Gislason, Phys. Rev., A 43, 4581 (1991).

[12] K. Urbanowski, Eur. Phys. J. C, 58, 151 (2008).

[13] K. Urbanowski, Cent. Eur. J. Phys., 7, 696 (2009).

[14] N. S. Krylov, V. A. Fock, Zh. Eksp. Teor. Fiz., 17, 93 (1947) [in Russian]. V. A. Fock, Fundamentals of Quantum mechanics, Mir Publishers, Moscow 1978.

[15] N. G. Kelkar, M. Nowakowski, J. Phys. A: Math. Theor., 43, 385308 (2010).

[16] J. Martorell, J. G. Muga, and D. W.L. Sprung, Lect. Notes Phys., 789, 239 - 275, (2009), (Springer-Verlag, Berlin, Heidelberg 2009).

[17] E. Torrontegui, J. G. Muga, J. Martorell, and D. W. L. Sprung, Advances in Quantum Chemistry, 60, 485 (2010). 
[18] Gaston Garcia-Calderon, Roberto Romo and Jorge Villavicencio, Physical Review B, 76, 035340 (2007).

[19] F. Giraldi, Eur. Phys. J. D., 69: 5, (2015).

[20] F. Giraldi, Eur. Phys. J. D., 70: 229, (2016).

[21] F. Giacosa, Found. of Phys., 42, 1262 (2012).

[22] F. Giacosa,Acta Phys. Polon. B, 47, 2135 (2016).

[23] M. L. Goldberger, K. M. Watson, Collision theory, Wiley 1964.

[24] R. E. A. C. Paley, N. Wiener, Fourier transforms in the complex domain, American Mathematical Society, New York, 1934.

[25] K. Urbanowski, Phys. Rev., A 50, 2847 (1994).

[26] K. Urbanowski, International Journal of Modern Physics A, 6, 1051 (1991).

[27] K. Urbanowski, International Journal of Modern Physics A, 7, 6299 (1992).

[28] K. Urbanowski, International Journal of Modern Physics A, 10, 1151 (1995).

[29] K. Urbanowski, International Journal of Modern Physics A, 13, 965 (1998). B 25, (1975), 739, and refernces therein.

[30] K.Urbanowski, International Journal of Modern Physics A, A8, 3721 (1993).

[31] NIST Handbook of Mathematical Functions, Natl. Inst.. Stand. and Technology, eds. F. W. J. Olver, D. W. Lozier, R. F. Boisvert and Ch. W. Clark,(Cambridge, 2010).

[32] K. Urbanowski, Eur. Phys. J. D, 71: 118, (2017); DOI: 10.1140/epjd/e2017-70666-0.

[33] M. Nowakowski, N. G. Kelkar, Nishiharima 2004, Penataquark - Proceedings of International Workshop on PENATAQUARK 04 (Spring 8, Hyogo, Japan, 23 -24 July 2004), pp. $182-189$. 
[34] M. Nowakowski, N. G. Kelkar, AIP Conf. Proc. 1030, 250 (2008); ArXiv: 0807.5103.

[35] V. Canuto and S. H. Hsieh, Phys. Rev. Lett. 39, 429 (1977).

[36] Y. K. Lau and S. J. Prokhovnik, Aust. J. Phys., 39, 339 (1986).

[37] M. S. Berman, Phys. Rev. D 43, 1075 (1991).

[38] J. L. Lopez and D. V. Nanopoulos, Mod. Phys. Lett. A 11, 1 (1996).

[39] L. M. Krauss and J. Dent, Physical Review Letters, 100, 171301 (2008).

[40] L. M. Krauss, J. Dent, and G. D. Starkman, Int. J. Mod. Phys. D 17, 2501 (2008). 